\title{
AVALIAÇÃO DE DESEMPENHO AMBIENTAL NA VALORIZAÇÃO DE RESÍDUOS SÓLIDOS DE PROCEŞSOS INDUSTRIAIS
}

\author{
ENVIRONMENTAL PERFORMANCE \\ ASSESSMENT OF SOLID WASTE RECOVERY' \\ FROM INDUSTRIAL PROCESS
}

Recebido: 04-03-2013

Aceite: $22-02-2014$

Mario Roberto dos Santos ${ }^{1}$

Cláudia Echevenguá Teixeira²

Cláudia Terezinha Kniess ${ }^{3}$

\section{RESUMO}

O objetivo deste estudo foi verificar, na literatura, as pesquisas relacionadas ao uso de resíduos sólidos industriais como matéria-prima alternativa na obtenção de novos materiais, bem como os métodos utilizados para estabelecer o seu desempenho ambiental. Foram consultadas as bases de dados Scopus, Scielo e Web of Science, no período de 2000 a 2012, resultando em 67 artigos que foram classificados em avaliação técnica (50) e avaliação ambiental (17). Os 50 artigos que avaliaram tecnicamente a utilização de resíduos foram descritivos e abordaram temas que podem ser agrupados em cinco grupos: redução de impactos e benefícios ambientais; redução e reaproveitamento de resíduos; uso de recursos naturais; ganhos econômicos; e outros temas. Dos 17 artigos que avaliaram os impactos da utilização desses resíduos, oito aplicaram o método de avaliação do ciclo de vida (ACV). Verificou-se que o emprego desse método varia muito, sem ser possível definir uma estrutura uniformizada quanto à valorização de resíduos na obtenção de novos materiais. Dessa forma, muitas vezes, é difícil identificar os benefícios associados aos métodos convencionais (materiais sem os resíduos) e informar o consumidor final ou usuário de um produto sobre as vantagens ambientais e econômicas.

Palavras-chave: Avaliação do ciclo de vida (ACV), Desempenho ambiental, Ecoeficiência, Resíduos sólidos.

1 Possui graduação em Engenharia pela Escola de Engenharia Mauá. Mestrado em Ciências Contábeis pela Fundação Escola de Comércio Álvares Penteado - FECAP. Atualmente é doutorando em Administração pela Universidade Nove de Julho - UNINOVE. São Paulo, São Paulo. Brasil. E-mail: mario.rsantos@terra.com.br

2 Possui graduação em Biologia pela Universidade de Caxias do Sul - UCS. Mestrado em Engenharia Civil pela Universidade Estadual de Campinas - UNICAMP. Doutorado em Engenharia Civil pela Université de Sherbrooke. Atualmente é professora em Administração na Universidade Nove de Julho e pesquisadora do Instituto de Pesquisas Tecnológicas - IPT - UNINOVE. São Paulo, São Paulo. Brasil. Email: ceteixeira10@gmail.com.

3 Possui graduação em Química pela Universidade Federal de Santa Catarina -UFSC. Mestrado em Engenharia Química pela Universidade Federal de Santa Catarina - UFSC e doutorado em Ciência e Engenharia de Materiais pela Universidade Federal de Santa Catarina - UFSC. Atualmente é professora na Universidade Nove de Julho - UNINOVE. São Paulo, São Paulo. Brasil. E-mail: kniesscl@ yahoo.com.br 


\section{ABSTRACT}

The aim was to investigate the literature, the research related to the use of industrial solid waste as raw material alternative to obtain new materials and methods to establish their environmental performance. The survey was conducted by consulting the databases: Scopus, Web of Science and Scielo, in the period 2000 - 2012 and found 67 articles. The articles were classified into technical (50) and environmental assessment (17). The 50 articles that have been technically assessment and descriptive themes can be grouped into five groups: reduced environmental impacts and benefits, reduction and reuse of waste, use of natural resources, economic gains, and other topics. The 17 articles that evaluated the impacts of using these residues, eight of them applied the life cycle assessment (LCA) method. It was found that the use of this method varies greatly, without being possible to define a uniform structure in the context of waste in obtaining new materials. Thus, it is often difficult to identify the associated potential benefits over conventional methods (materials without waste) and inform final consumer or user of a product and the environmental benefits and which are, therefore, also represent an economic advantage.

Keywords: Life cycle assessment (LCA), Environmental performance, Eco-efficiency, Solid waste.

\section{INTRODUÇÃO}

A sociedade e os processos produtivos industriais geram cada vez mais resíduos sólidos, líquidos e gasosos, os quais, muitas vezes, são descartados sem controle, ocasionando impactos ambientais de consequências e graus variáveis (ALLWOOD et al., 2011; FLEISCHMANN et al., 1997). Com a crescente concorrência global, aliada a discussões acerca da sustentabilidade, notase que, em concomitância com a busca por uma maior eficiência da produção, ocorre gradativamente a incorporação de aspectos para redução de impactos ambientais. Segundo Pinsky, Dias e Krugliankas (2013, p. 465), "o aumento da importância da sustentabilidade nos últimos anos tem levado algumas empresas a considerar, como parte integrante da estratégia de negócios, a inclusão de metas empresariais compatíveis com o desenvolvimento sustentável". Contudo, ainda muitos desafios precisam ser superados no campo político, social, econômico e técnico para equacionar o problema da geração, da coleta, do transporte e do tratamento de resíduos (LIAMSANGUAN; GHEEWALA, 2008).

Evitar o desperdício é um conceito intuitivo que tem estado presente na sociedade ao longo do tempo. Desvio de recursos, perdas econômicas e poluição ambiental têm sido reconhecidos como as consequências da geração de resíduos, mas a extensão e o impacto de tais consequências estão apenas começando a ser compreendidos no contexto da sustentabilidade. Nesse contexto, a redução de resíduos deve ser analisada como uma iniciativa de negócio auto-orientada, independentemente de incentivos governamentais e regulamentações (BAUTISTA-LAZO; SHORT, 2013).

A reciclagem é considerada ambientalmente benéfica e favorável ao desenvolvimento econômico sustentável, pois atenua o uso de novos recursos, diminui a demanda por espaços nos aterros e, geralmente, envolve redução no consumo de energia. Além disso, a coleta de materiais para reciclagem tem seus próprios impactos ambientais, principalmente no que se refere à energia utilizada na coleta e triagem e à utilização dos materiais recuperados em novos produtos (CRAIGHILL; POWELL, 1996).

A necessidade da utilização sustentável dos recursos naturais é um tema amplamente discutido, mas não há consenso sobre uma definição clara de como deve ser esse uso nem de como deve ser medido. Dependendo da definição utilizada, pode compreender apenas o consumo de matéria-prima ou o consumo e a poluição emitida pelo uso desses recursos. Por conseguinte, uma quantidade enorme de indicadores pode ser aplicada, de modo que o resultado de um estudo de avaliação do ciclo de vida com o objetivo de quantificar a utilização de recursos 
depende da seleção de categorias de impacto avaliadas (BERGER; FINKBEINER, 2011).

Na construção de comunidades sustentáveis, por exemplo, é necessário considerar os efeitos indiretos (da aquisição de matérias-primas, da produção, do transporte e da conclusão) e os efeitos diretos (da fabricação em si e do uso) dos ciclos de vida de produtos e serviços (Yl et al., 2007). Assim, na tentativa de absorver os benefícios de um material e na busca de soluções para a diminuição de seu custo, a introdução de matérias-primas alternativas constitui um importante aspecto a ser pesquisado. A reutilização de certos materiais considerados resíduos permite que estes retornem ao ciclo de processamento para uso posterior, otimizando a relação energia/meio ambiente/materiais, o que acarreta um melhor aproveitamento dos recursos, sejam eles materiais ou energéticos. No entanto, é necessário um alto nível de tecnologia e de desenvolvimento desses materiais a fim de poderem competir com os materiais convencionais (ESTRELA, 1996).

Uma das formas de avaliar os possíveis benefícios ou impactos ambientais decorrentes da reutilização de resíduos em processos produtivos é por meio do método de avaliação do ciclo de vida (ACV) do produto. Apesar disso, descobriu-se que diferentes ACV's de um mesmo produto podem apresentar resultados diferentes, o que levou a uma discussão entre especialistas e usuários sobre o que é correto e o que não é em uma ACV (STEEN, 2006).

Dentro desse contexto, o objetivo geral deste estudo foi verificar na literatura, por intermédio de uma revisão sistemática, as pesquisas relacionadas ao uso de resíduos sólidos industriais como matéria-prima alternativa na obtenção de novos materiais e os métodos utilizados para estabelecer o seu desempenho ambiental. Além disso, buscou-se verificar a utilização de resíduos sólidos industriais na indústria cerâmica e de resíduos provenientes da combustão de carvão mineral nas indústrias em geral.

Para isso, este trabalho está estruturado da seguinte forma: após esta introdução, a seção dois apresenta o referencial teórico utilizado sobre ACV e avaliação de ecoeficiência; na seção três, consta o método de pesquisa utilizado; na seção quatro, estão os resultados alcançados; e, na seção cinco, expõem-se as considerações finais.

\section{REFERENCIAL TEÓRICO}

Nesta seção, serão apresentadas algumas definições sobre geração de resíduos, gestão de resíduos e ACV.

\subsection{Geração de resíduos}

Resíduos sólidos são definidos pela NBR 10000:2004 da Associação Brasileira de Normas Técnicas (ABNT) como "[...] resíduos nos estados sólido e semissólido, que resultam de atividades de origem industrial, doméstica, hospitalar, comercial agrícola, de serviços e de varrição" (ABNT, 2004, p. 1). Essa norma classifica os resíduos sólidos, quanto aos seus riscos potenciais ao meio ambiente e à saúde pública, em dois grupos: resíduos classe I - Perigosos; e resíduos classe II - Não-perigosos, os quais são subdivididos em resíduos classe II A - Não-inertes e resíduos classe II B - Inertes. A classificação é baseada na caracterização do resíduo em função das matérias-primas, dos insumos e do processo que the deram origem.

A geração de resíduos sólidos é um dos graves problemas da atualidade em razão do crescimento gradativo e desordenado populacional, da aceleração do processo de ocupação do solo (VALERIO; SILVA; COHEN, 2008) e do crescimento acentuado da descartabilidade dos bens de consumo. Nesse contexto, surge a Política Nacional de Resíduos Sólidos (PNRS), instituída pela lei 
n. 12.305, de 02 de agosto de 2010, e regulamentada pelo decreto n. 7.404 , de 23 de dezembro de 2010 em seu artigo 13, que estabelece duas categorias de classificação de resíduos: I - quanto à origem (domiciliares, de limpeza urbana, urbanos, de estabelecimentos comerciais, de serviços públicos, industriais, de serviços de saúde, da construção civil, agrossilvopastoris, de serviços de transportes e de mineração) e II - quanto à periculosidade (perigosos e não perigosos) (PNRS, 2010).

A pesquisa de Jacobi e Besen (2011) oferece uma visão abrangente acerca da origem dos resíduos, em razão das atividades que os geram, conforme é mostrado no Quadro 1.

\begin{tabular}{|l|l|l|} 
Público & Varrição e poda & Folhas, galhos de arvores, papéis etc. \\
\hline Serviços de saúde & $\begin{array}{l}\text { Hospitais, clínicas, } \\
\text { consultórios, laboratórios etc. }\end{array}$ & $\begin{array}{l}\text { Grupo A - biológicos: sangue, tecidos, vísceras, resíduos de } \\
\text { análises clínicas etc. Grupo B - químicos: medicamentos vencidos e } \\
\text { interditas, termômetros, objetos cortantes etc. Grupo C - } \\
\text { radioativos. Grupo D - comuns, não contamindos, papeis, plásticos, } \\
\text { vidros, embalagens etc. }\end{array}$ \\
\hline Industrial & $\begin{array}{l}\text { Cinzas, lodos, efluentes liquidos, adsorventes, catalizadores, } \\
\text { poeiras, óleos, resíduos alcalinos ou ácidos, escórias, plásticos, } \\
\text { papel, madeira, fibras etc }\end{array}$ \\
\hline $\begin{array}{l}\text { Portos, aeroportos, } \\
\text { terminais }\end{array}$ & Portos, aeroportos e terminais & $\begin{array}{l}\text { Resíduos sépticos, sobras de alimentos, material de higiene e asseio } \\
\text { pessoal etc. }\end{array}$ \\
\hline Agrícola & $\begin{array}{l}\text { Embalagens de agrotóxicos, pneus e óleos usados, embalagens de } \\
\text { medicamentos veterinários, recipientes, plásticos etc. }\end{array}$ \\
\hline Construção civil & $\begin{array}{l}\text { Obras e reformas residencias } \\
\text { e comerciais }\end{array}$ & $\begin{array}{l}\text { Madeira em geral, cimento, blocos, pregos, gesso, tinta, latas, } \\
\text { cerâmicas, pedra, areia, terra etc. }\end{array}$ \\
\hline
\end{tabular}

Quadro 1 - Classificação de resíduos

Fonte: adaptado de Jacobi e Besen (2011).

Outra forma de classificar a geração de resíduos é a adotada por Leite (2009), que leva em consideração o fluxo de retorno, isto é, o fluxo dos canais reversos de distribuição dos produtos após o seu uso:

a) pós-consumo - referente aos bens que, após seu ciclo de vida útil, são descartados de diferentes maneiras, constituindo os produtos de pós-consumo e que podem ter potencial para reutilização;

b) pós-venda - refere-se aos bens industriais de pós-venda, caracterizados por apresentarem pouco ou nenhum uso e que retornam à cadeia de suprimentos por diversos motivos, tais como retorno de produtos em consignação, embalagens retornáveis, ajuste ou excesso de estoques, devoluções em vendas diretas ao consumidor final, atendimentos de reclamações sobre qualidade ou defeito ou erro de expedição, garantia ou qualidade por defeitos de fabricação ou de funcionamento; avarias no produto ou na embalagem e outros aspectos de qualidade intrínseca aos produtos vendidos e substituição de componentes.

O crescimento do pós-consumo é determinado pelo aumento de lançamentos de novos produtos e pelo uso de outras fontes de materiais constituintes, em casos em que os metais são substituídos por plásticos. Esse fenômeno, segundo Leite (2009), pode ser observado mais intensamente no setor automobilístico e de tecnologia da informação, em que há um crescimento demasiado na produção de acessórios e periféricos. Esses resíduos podem ter três destinos diferentes: um local de descarte seguro, como aterros sanitários e depósitos específicos; um destino não seguro, sendo lançado na natureza, o que acarreta a poluição do ambiente; ou voltar a uma cadeia de produção.

Alguns setores merecem destaque quanto à geração de resíduos e de impactos sanitários e ambientais, como, por exemplo: (i) pneus inservíveis devido ao volume acumulado (ALBUQUERQUE NETO et al., 2005; REDA TAHA et al., 2008); (ii) construção civil, que consome grandes 
quantidades de energia, recursos naturais e materiais e gera níveis inaceitáveis de resíduos sólidos (YUAN et al., 2012); (iii) equipamentos elétricos e eletrônicos, que são um dos maiores utilizadores de metais e, com isso, tornaram-se fonte de geração de resíduos (OGUCHI et al., 2012) e uma das principais causas do esgotamento de recursos naturais (WONG; LEE; YUNG, 2010).

Os resíduos provenientes dos processos produtivos ou industriais ocupam também uma posição de destaque devido às crescentes quantidades de resíduos e subprodutos industriais que geram. Além disso, conforme Castro-Gomes et al. (2012), na maioria dos países da União Europeia (UE), é o principal setor econômico responsável pelo fato.

Nesse contexto, a gestão de resíduos é uma atividade que vem ganhando inportância na atualidade, por se tornar uma fonte geradora de matéria-prima alternativa e, ao mesmo tempo, por contribuir para a redução do uso dos recursos naturais. Com a finalidade de minimizar o descarte de substâncias na natureza e de reduzir o consumo de recursos naturais, tem havido muito empenho no sentido de reintegrar os resíduos nos processos produtivos, por meio, por exemplo, da reciclagem, do reuso e da recuperação, permitindo, assim, um desenvolvimento sustentável e a redução de passivos ambientais.

\subsection{Gestão de resíduos}

O objetivo de reduzir o impacto ambiental da atividade industrial é amplamente aceito como uma meta a ser alcançada, fazendo com que muitas empresas se orgulhem de ter produtos "verdes" e práticas de negócios "sustentáveis". Na fabricação, essas práticas de negócios podem variar, incluindo, por exemplo, a substituição de materiais não biodegradáveis por biodegradáveis, a reciclagem de produtos e a redução nas cadeias de suprimentos do consumo de energia e das emissões atmosféricas. A implementação dessas práticas empresariais é feita com maior profundidade quando a redução dos impactos ambientais está associada ao aumento dos lucros para o negócio como um todo (HODGE; OCHSENDORF; FERNÁNDEZ, 2010).

Ressalta-se, ainda, que benefícios ambientais significativos podem ser obtidos por meio de diferentes processos de gestão de resíduos, conforme preconizam Ekvall et al. (2007):

a) incineração de resíduos com recuperação de energia reduz a necessidade de outras fontes de energia;

b) materiais provenientes de processos de reciclagem substituem a necessidade de produção de material virgem;

c) tratamento biológico pode reduzir a necessidade de produção de fertilizantes artificiais e combustíveis para veículos.

Segundo Fiksel et al. (2011), como é crescente a preocupação com a sustentabilidade das cadeias de suprimentos industriais, os organismos governamentais e o setor privado estão elevando os esforços para melhorar a gestão dos resíduos sólidos. Tal situação decorre do fato de que a disposição em aterros sanitários tem sido o principal método de gestão de resíduos domiciliares e industriais nas últimas décadas em muitos países, gerando, assim, grandes problemas ambientais (Di BELLA et al., 2012; LANER et al., 2012). Mesmo alguns países altamente industrializados, tais como os Estados Unidos da América (EUA), a Austrália, o Reino Unido e a Finlândia, têm uma dependência muito grande do uso de aterros (LANER et al., 2012).

Objetivando-se a minimização de descarte de substâncias na natureza e também a redução do consumo de recursos naturais, têm-se buscado reintegrar os resíduos nos processos produtivos originais, seja por reciclagem, reuso ou recuperação, a fim de permitir desenvolvimento sustentável e redução de passivos ambientais ou destinação desses resíduos a outros processos de 
produção. Tendo isso em vista, o estudo de Bautista-Lazo e Short (2013) busca constituir um modelo para entender o impacto e uso potencial de resíduos com base no conceito da simbiose industrial. Chertow (2000) explicita que a simbiose industrial envolve a troca de materiais, energia, água ou subprodutos entre indústrias tradicionalmente separadas. Segundo Jacobsen (2006), esses relacionamentos implicam alguns benefícios econômicos diretos e indiretos, constituindo uma forma interessante para a colaboração interindustrial, e resultam em diminuição dos danos ambientais.

De acordo com Bautista-Lazo e Short (2013), perdas econômicas e poluição ambiental são reconhecidas como as consequências do desperdício, mas a extensão e o impacto de tais consequências estão apenas começando a serem entendidos no contexto da sustentabilidade. A eliminação dos resíduos é, assim, um dos pilares da competitividade empresarial, que, devido ao aumento da consciência ambiental, tem se tornado cada vez mais crucial para os fabricantes. Nesse contexto, a redução de resíduos deve ser vista como uma autoiniciativa de negócio, independentemente de incentivos e regulamentações governamentais.

Bautista-Lazo e Short (2013) definem como saídas de um processo produtivo de uma empresa: (i) produtos e subprodutos, incluindo as embalagens, sendo considerado subproduto tudo o que é produzido incidentalmente durante a fabricação do produto principal; e (ii) não produtos, nos quais estão incluídos os resíduos sólidos, as águas residuais e as emissões atmosféricas. A Figura 1 apresenta o modelo desenvolvido pelos autores de acordo com a perspectiva denominada por eles de "the all seeing eye of business".

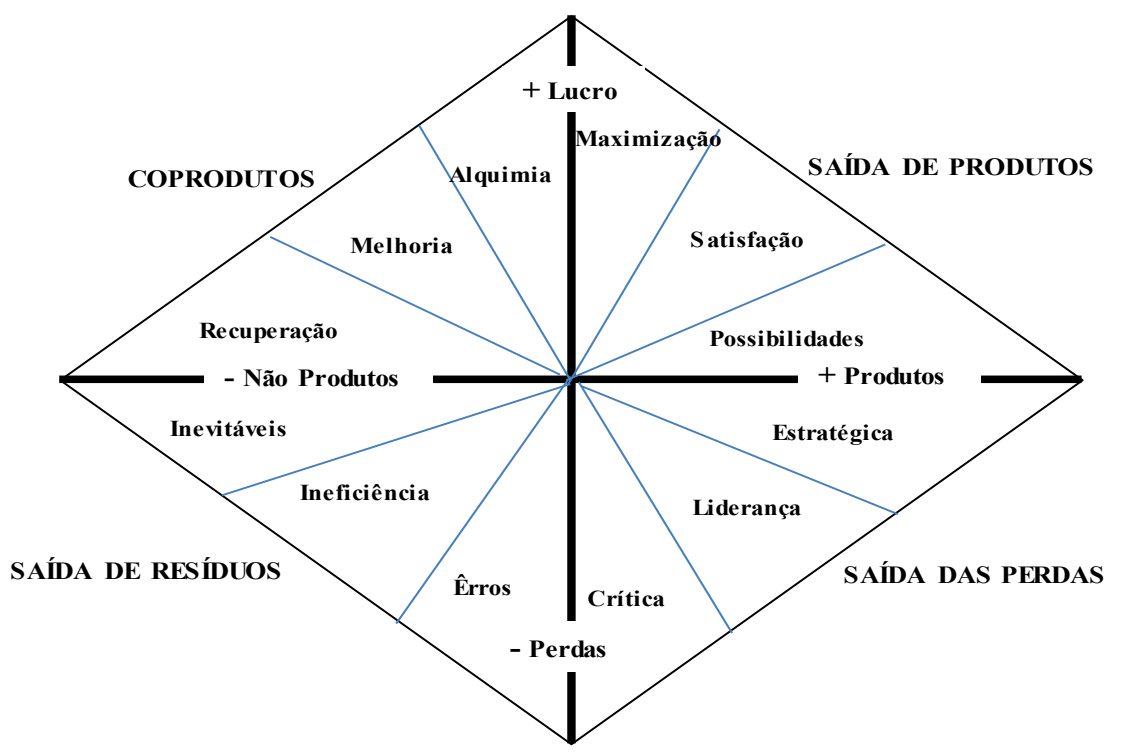

Figura 1 - "The all seeing eye of business"

Fonte: adaptado de Bautista-Lazo e Short (2013).

Transformar resíduos em coprodutos tem algumas ressalvas, pois melhoramentos ambientais somente são obtidos se o processo de transformação for mais eficiente do que o uso de matérias -primas virgens, existindo, portanto, a necessidade de uma avaliação do ciclo de vida completo para cada transformação de resíduos. Às vezes, em razão do nível da tecnologia utilizada, pode-se revelar como inviável a eliminação completa da geração de resíduos (BAUTISTA-LAZO; SHORT, 2013). 


\subsection{Avaliação de Ciclo de Vida (ACV)}

A ACV é um método de gestão ambiental aplicável a um produto, entendendo-se produto como um bem ou um serviço. Esse método de gestão ambiental se dedica a avaliar todos os estágios de vida de um produto, desde a origem dos recursos no meio ambiente até a disposição final dos resíduos de materiais após o uso. Trata-se de uma visão holística que passa, também, por todas as etapas intermediárias, como beneficiamento, transporte e estocagem, isto é, do berço ao túmulo (cradle to grave) (BARBIERI, 2011; FINNVEDEN et al., 2009; REBITZER et al., 2004). As normas que norteiam esse método (ISO 14040 e 14044) são consideradas o principal e mais importante padrão internacional de avaliação ambiental e são usadas como referências em outros processos, como, por exemplo, a pegada de carbono (ISO 14067) e a ecoeficiência (ISO 14045) (KLÖPFFER, 2012).

O método ACV é descrito, no Brasil, pela norma NBR ISO 14040 - Gestão Ambiental Avaliação do ciclo de vida - Princípios e estrutura (ABNT, 2009a) e pela norma NBR ISO 14044 - Gestão Ambiental - Avaliação do ciclo de vida - Requisitos e orientações (ABNT, 2009b). Essas normas definem ACV como a compilação e avaliação das entradas, das saídas e dos impactos ambientais potenciais de um sistema de produto durante o seu ciclo de vida (ABNT, 2009a).

Segundo Contreras et al. (2009), uma das mais importantes aplicações de uma ACV é a análise das contribuições para o impacto ambiental como um todo de um determinado produto, normalmente com a finalidade de melhorá-lo ou compará-lo com similares, visando tanto às comunicações internas quanto às externas (mercado, orgãos reguladores etc.). Para que a ACV seja uma fonte eficiente de apoio à tomada de decisão, é crucial, para sua elaboração, o uso de informações e de dados confiáveis apresentados no inventário do ciclo de vida (ICV). Uma ACV de produtos e serviços é muito dependente de bancos de dados confiáveis e abrangentes que complementem o processo de fabricação, operação, desmontagem e gestão de resíduos (FRISCHKNECHT, 2006). Guinée et al. (2002) alertam que os impactos ambientais são frequentemente descritos como impactos potenciais, porque eles não são especificados no tempo e no espaço e estão relacionados a determinada unidade funcional que é, muitas vezes, definida arbitrariamente.

A caracterização da modelagem das emissões de substâncias em uma avaliação de impactos do ciclo de vida (AICV) comumente usada é tipicamente focada em propriedades inerentes às substâncias, enquanto o ambiente é representado como uma unidade global padronizada, com características genéricas ou médias, e pode ser modelado de maneira simplificada (POTTING; HAUSCHILD, 2006). Segundo Jolliet (2006), a AICV emergiu da conjunção de três fatores principais:

a) a crescente necessidade de melhores informações ambientais para tomada de decisão na fabricação, na utilização e na disposição de produtos;

b) o desenvolvimento das ciências ambientais com novos conhecimentos e modelos, identificando os mecanismos envolvidos nos problemas ambientais;

c) um grupo de pioneiros provenientes de horizontes diferentes, mas com uma visão comum e um senso de urgência, percebeu a necessidade de superar a enorme lacuna entre a tomada de decisão e a questão dos resíduos, sendo preciso considerar a ciência ambiental para compreender e avaliar de que modo a decisão sobre os produtos afeta as emissões e, eventualmente, o meio ambiente.

Tendo em vista o objetivo da avaliação de impactos, a International Organization for Standardization (ISO) editou a norma ISO 14045: Environmental management - Eco-efficiency assessment of product systems - Principles, requirements and guidelines, com primeira edição em 15 de maio de 2012, ainda sem uma norma correspondente na ABNT, que criou uma metodo- 
logia de avaliação de ecoeficiência. A norma brasileira correspondente é a ABNT NBR ISO 14045: Gestão ambiental - Avaliação da ecoeficiência de sistemas de produto - Princípios, requisitos e orientações, com primeira edição em 21 de maio de 2014. A avaliação de ecoeficiência é uma metodologia quantitativa de gestão que permite o estudo de impactos ambientais durante o ciclo de vida de um sistema de produto junto com o seu sistema de valor (ISOABNT 14045, 20124). Os impactos ambientais são avaliados utilizando a ACV, conforme prescrito pelas outras normas (ISOABNT 14040 e ISO 14044), compartilhando, assim, muitos princípios, tais como perspectiva de ciclo de vida, abrangência, abordagem de unidade funcional, natureza iterativa, transparência e prioridade para uma abordagem científica.

As AICVs podem ser avaliadas por diferentes métodos, como descrito por Pizzol (2011): CML 2001; Eco-Indicator 1999; IMPACT 2002; ECOPOINTS 97; EPS 2000; EDIP 2003; TRACl; ReCiPe; STEPWISE 2006 e USEtox. Pode-se acrescentar, também, os métodos LIME (ITSUBO; INABA, 2003) e LUCAS (TOFFOLETTO et al., 2007).

Esses métodos são classificados em duas categorias (JOLLIET et al., 2003): (i) métodos clássicos - que restringem uma modelagem quantitativa às fases iniciais na cadeia de causa-efeito, com o objetivo de limitar as incertezas (eg. CML 2001 e EDIP 2003), em que os resultados da ICV são apresentados em categorias de ponto médio (midpoint), como, por exemplo, alterações climáticas ou ecotoxicidade; e (ii) métodos orientados ao dano - como Eco-Indicator 99 ou EPS 2000, que tentam modelar a cadeia de causa e efeito até o ponto final (endpoint), isto é, onde ocorre o impacto ambiental, às vezes com grandes incertezas.

\section{MÉTODO}

O levantamento dos artigos referentes à valorização de resíduos sólidos utilizados em estudos de incorporação em produtos como substitutos de matérias-primas originais foi baseado no método de revisão bibliográfica sistemática (BRERETON et al., 2007). A revisão sistemática é uma metodologia realizada em três fases - (i) definição do plano de revisão; (ii) execução da revisão; e (iii) emissão do documento de revisão - e tem o objetivo de fornecer um resumo equilibrado do que é relevante para atender uma necessidade específica de informação (BRERETON et al., 2007).

Ressalta-se, ainda, que a revisão da literatura é uma característica essencial de qualquer projeto acadêmico, pois cria uma base sólida para o avanço do conhecimento e facilita o desenvolvimento da teoria, além de descobrir áreas em que a pesquisa é necessária (WEBSTER; WATSON, 2002). Em uma revisão sistemática de literatura, inicialmente, faz-se uma pesquisa abrangendo todas as palavras-chave definidas para a busca e, posteriormente, submetem-se os artigos encontrados a um refinamento a fim de avaliar somente os que se enquandram no objeto estudado, descartando-se os demais.

Esta pesquisa foi realizada por meio de consulta a bases de dados relevantes dentro da área acadêmica: Scopus, Scielo e Web of Science. Artigos acadêmicos, publicados no período de 2000 a 2012 foram pesquisados. A avaliação priorizou os estudos voltados à valorização/utilização de resíduos sólidos resultantes de processos produtivos industriais e também o uso de cinzas de carvão mineral.

As seguintes palavras-chave foram utilizadas na busca: waste; reuse; recycling; coal ash; coal botton ash; life cycle assessment review; cinzas; reuso; reciclagem de resíduos sólidos; cinzas pesadas; cinzas de carvão; avaliação de resíduos; avaliação de ciclo de vida. As palavras waste, reuse, recycling, coal ash e botton ash foram agrupadas com o intuito de reduzir o universo da pesquisa: waste recycling reuse; coal ash reuse e coal botton ash.

Após a leitura dos títulos (se estes se enquadrassem no objetivo deste estudo), foram 
lidos os resumos/abstracts e, finalmente, o artigo completo. Depois dessa verificação, foram selecionados os artigos que tratavam da reutilização de resíduos sólidos provenientes de processos industriais. Foram descartados, por exemplo, casos em que o tema consistia na reutilização de resíduos sólidos: urbanos provenientes de aterros sanitários, pós-consumo e para geração de energia.

\section{RESULTADOS}

A pesquisa nas três bases de dados resultou em um total de 1709 artigos, dos quais foram selecionados 67 artigos, listados na Tabela 1. Percebe-se, com base no número de publicações no periódico Cerâmica, que é especializado na divulgação de trabalhos científicos sobre a indústria cerâmica, um grande interesse no uso de resíduos industriais nesse ramo.

Tabela 1 - Periódicos e respectivas quantidades de artigos encontrados

\begin{tabular}{l|c}
\hline Periódicos & $\begin{array}{c}\text { Quantidade de } \\
\text { Artigos }\end{array}$ \\
\hline Cerâmica & 27 \\
Journal of Cleaner Production & 13 \\
Resources, Cons ervation and Recycling & 6 \\
Journal of Environmental Management & 4 \\
Journal of Hazardous Materials & 4 \\
International Journal of Life Cycle Assessment & 4 \\
Building and Environment & 2 \\
Applied Clay Science & 1 \\
Chemical Engineering Journal & 1 \\
Construction and Building Materials & 1 \\
Environmental Science \& Tecnology & 1 \\
Hydrometallurgy & 1 \\
Revista Matéria & 1 \\
Waste Management \& Research & 1 \\
Total & 67 \\
\hline
\end{tabular}

O Gráfico 1 apresenta a evolução da publicação de artigos sobre a utilização de resíduos sólidos industriais como matéria-prima no período pesquisado.

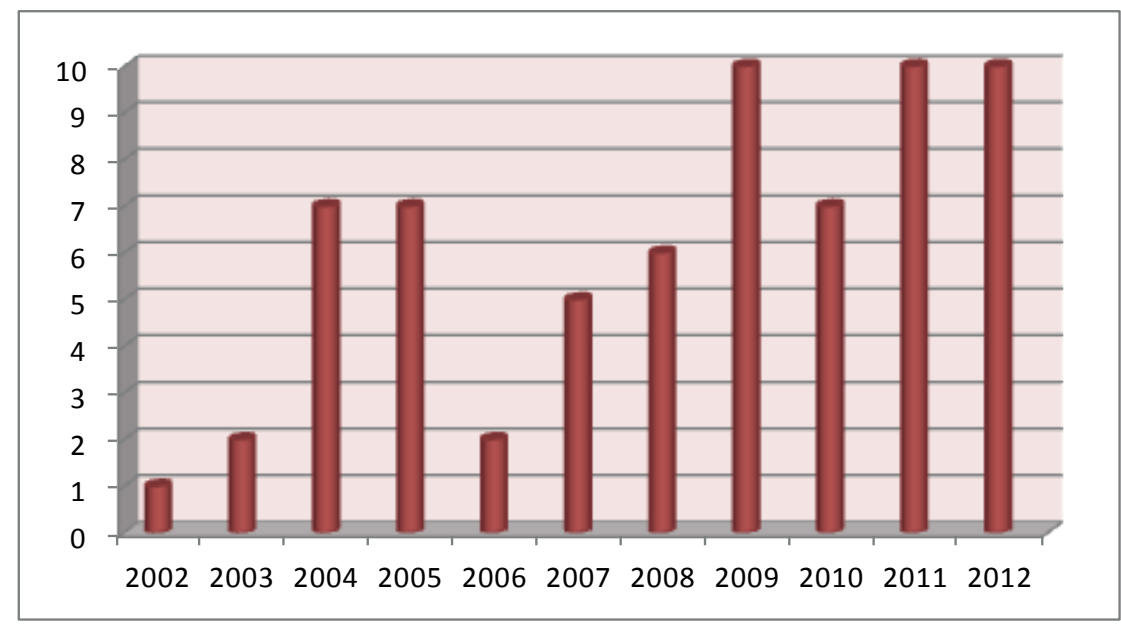

Gráfico 1 - Quantidade de artigos publicados por ano 
Esse gráfico mostra que o interesse por esse tipo de pesquisa vem aumentando, provavelmente em razão do aumento da produção dos bens de consumo e da consequente geração de resíduos industriais. Essa afirmação é corroborada por Campos et al. (2007), que citam que a inserção dos resíduos industriais em outros ciclos de produção deve ser avaliada como uma opção real de reaproveitamento tanto da perspectiva ambiental quanto da econômica.

Os 67 artigos encontrados foram classificados em artigos técnicos (50) e artigos ambientais (17). Os artigos técnicos são aqueles que não fazem uma avaliação ambiental do uso dos resíduos, mas somente descrevem as vantagens e desvantagens de tal uso. Já os artigos ambientais fazem uma avaliação ambiental, descrevendo os métodos e critérios utilizados. Essas avaliações serão discutidas a seguir.

\subsection{Artigos técnicos}

A Tabela 2 mostra os resíduos industriais avaliados nas pesquisas, o processo produtivo no qual seriam utilizados e as quantidades de artigos que os estudaram.

Tabela 2 - Resíduos sólidos industriais e sua utilização como matéria-prima em outros produtos

\begin{tabular}{|c|c|c|}
\hline Resíduo indus trial & Produtos & Artigos \\
\hline $\begin{array}{l}\text { Granito, mármore e rocha } \\
\text { gnaisse }\end{array}$ & Cerâmicos: porcelana; blocos; ladrilhos vitrificados e cerâmica em geral & 10 \\
\hline Cerâmica & $\begin{array}{l}\text { Isoladores elétricos; argamassas; concreto; cimento Portland e uso no } \\
\text { próprio processo produtivo }\end{array}$ & 6 \\
\hline Siderúrgicos & $\begin{array}{l}\text { Produtos com argila; cerâmica; ferro; pigmento cerâmico e uso no } \\
\text { próprio processo produtivo }\end{array}$ & 6 \\
\hline Carvão mineral & $\begin{array}{l}\text { Uso geral (cimento, concreto; telhas etc.); corantes e adsorventes; } \\
\text { Zeolito sintético e geração de energia }\end{array}$ & 5 \\
\hline Caulim & Blocos e revestimentos cerâmicos; zeólita A e corpos mulíticos & 5 \\
\hline Bagaço de cana & Cerâmica vermelha (tijolos, telhas etc.) & 3 \\
\hline Construção cívil & Argamassa e utilização na própria na obra & 2 \\
\hline Tijolo & Tijolos e cerâmicas & 2 \\
\hline Celulose & Gesso & 1 \\
\hline Alumina & Blocos e telhas cerâmicas & 1 \\
\hline Alumínio & Utilização no próprio processo produtivo & 1 \\
\hline Areia de fundição & Reutilização no próprio processo & 1 \\
\hline Argila; gnaiss e e varvito & Cerâmica & 1 \\
\hline Carbonato de sódio & Sulfato de cálcio & 1 \\
\hline Casca de arroz & Mulita & 1 \\
\hline Cobre & Argamassas & 1 \\
\hline Minas de tungstênio & Revestimentos de construção & 1 \\
\hline Papel & Utilização no próprio processo produtivo & 1 \\
\hline \multirow[t]{2}{*}{ Silício } & Utilização no próprio processo produtivo & 1 \\
\hline & Total de Artigos & 50 \\
\hline
\end{tabular}

As avaliações desses artigos foram descritivas, e os temas abordados podem ser classificados em cinco grupos: redução de impactos e benefícios ambientais; redução e reaproveitamento de resíduos; uso de recursos naturais; ganhos econômicos; e outros temas, descritos a seguir:

a) redução de impactos e possíveis benefícios ambientais foi o fator mais mencionado pelos autores, sendo a redução de impacto ambiental o tema dominante, seguido pela proteção ambiental, pela redução de poluição, pelos benefícios ambientais, pela redução de passivo ambiental etc.;

b) a redução e o reaproveitamento de resíduos englobaram temas como reaproveitamento para melhoria de processo industrial, matéria-prima, redução na quantidade de resíduos gerados, busca de alternativas viáveis, maximização do uso etc.;

c) o uso de recursos naturais englobou diminuição do consumo de recursos naturais, conservação e prolongamento das reservas de matérias-primas, redução do consumo de combustíveis e energia etc.; 
d) ganhos econômicos englobaram contribuição econômica, ganhos, redução e minimização de custos, custos de armazenamento e transporte de resíduos, economia no consumo de matérias-primas etc.;

e) outros temas mencionados foram desenvolvimento autossustentado, melhoria da imagem empresarial, minimização de prejuízos sociais, benefícios sociais, reutilização de solos ocupados com resíduos, entre outros.

Esses artigos somente mencionaram os possíveis resultados ambientais do processo de reuso ou reciclagem de resíduos industriais de uma forma intuitiva, mas não apresentaram possíveis valores analisados, sejam financeiros ou índices/fatores de desempenho ambiental, que pudessem suportar tais afirmações, já que reciclar/reusar nem sempre é a melhor solução para o reaproveitamento de um resíduo.

\subsection{Artigos com avaliação ambiental}

A Tabela 3 mostra a metodologia empregada na avaliação ambiental do uso dos resíduos sólidos industriais e os autores dos 17 artigos, bem como o ano e o país onde foi realizada a pesquisa. Pode-se verificar que o método ACV foi o mais utilizado (47\%), não havendo concentração dos artigos analisados em único país ou região.

Tabela 3 - Artigos e metodologias empregadas

\begin{tabular}{|c|c|c|}
\hline Emissão de gases "efeito estufa" (GHG) & $\begin{array}{l}\text { O’Brien, Ménaché e O'Moore } \\
\text { (2009)/Australia; Hodge, Ochsendorf e } \\
\text { Fernández (2010)/EUA; McLellan et al. } \\
\text { (2011)/Austrália }\end{array}$ & 3 \\
\hline Emissão de $\mathrm{CO}_{2}$ & Fröhling e Rentz (2010)/Alemanha & 1 \\
\hline $\begin{array}{l}\text { Poluição (resíduos e poluentes), consumo de água } \\
\text { potável, energia e custos }\end{array}$ & Hu et al. (2011)/China & 1 \\
\hline $\begin{array}{l}\text { Pegada de carbono, emissão de } \mathrm{CO}_{2} \text {, consumo de } \\
\text { energia e custos }\end{array}$ & Yang et al. (2011)/Singapura & 1 \\
\hline $\begin{array}{l}\text { Aquecimento global, acidificação, depleção abiótica, } \\
\text { camada de ozônio, toxidade humana e ecotoxidade da } \\
\text { água }\end{array}$ & Habert, Lacaillerie e Roussel (2011)/França & 1 \\
\hline Total de artigos & & 17 \\
\hline
\end{tabular}

O Quadro 2 mostra como os autores que utilizaram o método ACV avaliaram os impactos ambientais. O que se nota nessas avaliações é que não há uma uniformidade quanto aos itens avaliados, o que é explicado por Guineé et al. (2002), pois, segundo os autores, a escolha dos impactos a serem avaliados e a tecnologia a ser utilizada depende do objetivo e escopo da ACV ou por Contreras et al. (2009), que explicitam que o método de processos de modelagens de multi-saídas deixa um leque de escolhas possíveis, de modo que há um componente subjetivo que apresenta vantagens e desvantagens, fazendo com que a escolha dependa do objetivo específico do estudo. 


\begin{tabular}{|l|l|}
\hline Babbitt e Lindner (2008b) & Aquecimento global; formação de poluição; acidificação; toxidade humana; ecotoxidade. \\
\hline Contreras et al. (2009) & $\begin{array}{l}\text { Saúde humana (cancerígenos, efeitos respiratórios de componentes orgânicos e inorgânicos, } \\
\text { mudanças climáticas, radiação e camada de ozônio); qualidade do ecossis tema (ecotoxidade, } \\
\text { acidificação, eutrofização, uso do solo); uso de recurs os (minerais, combustíveis fósseis). }\end{array}$ \\
\hline Chowdhury, Apul e Fry (2010) & $\begin{array}{l}\text { Custos (de construção); consumo de energia; acidificação; aquecimento global; toxidade } \\
\text { humana; ecotoxidade aquática; sedimentos de ecotoxidade aquática; ecotoxidade do solo. }\end{array}$ \\
\hline Chen et al. (2010) & $\begin{array}{l}\text { Emissão p/ o ar (aquecimento, cinzas, HCl, Pb, Ni, Mn, Metano, NOx, H2S, CO, CO2, dioxinas). } \\
\text { Impactos: depleção abiótica; aquecimento global; depleção da camada de ozônio; toxidade } \\
\text { humana; ecotoxidade aquática; ecotoxidade marinha; ecotoxidade do solo; oxidação } \\
\text { fotoquímica; acidificação; eutrofização e consumo de energia. }\end{array}$ \\
\hline Prasara-A e Grant (2011) & $\begin{array}{l}\text { Mudanças climáticas; depleção da camada de ozônio; acidificação do solo; eutrofização da } \\
\text { água; eutrofização marinha; toxidade humana; oxidação fotoquímica; formação de partículas; } \\
\text { ecotoxidade do solo; ecotoxidade da água; ecotoxidade marinha; ocupação do solo agrícola; } \\
\text { transformação natural do solo; depleção da água, depleção de metais e depleção de fósseis. }\end{array}$ \\
\hline
\end{tabular}

Quadro 2 - Artigos com método ACV e fatores avaliados

Os autores Chowdhury, Apul e Fry (2010) analisaram em sua pesquisa os custos de transporte dos resíduos, comparando-os com a construção de um aterro para a sua disposição. Nota-se que, dentre as oito pesquisas, somente esses autores focaram, de alguma forma, os custos. Essa é uma das limitações mencionadas por Gäbel e Tillman (2004), para quem, sob o ponto de vista industrial, os modelos convencionais de ICV não abordam o desempenho do produto nem o custo econômico.

Dentre os artigos que não utilizaram a método ACV, dois artigos usaram testes de lixiviação (BRUNORI et al., 2005; OLIVEIRA; HOLANDA, 2004), realizados conforme as normas vigentes nos respectivos países (Itália e Brasil), o que não implica, necessariamente, uma mesma forma de avaliação, podendo acarretar diferentes resultados. Outros três artigos utilizaram as emissões de gases que provocam o efeito estufa (GHG) nas suas avaliações. Dois desses artigos - Hodge, Ochsendorf e Fernández (2010) e McLellan et al. (2011) - usaram custos como métrica de avaliação também. McLellan et al. (2011) alertam que os indicadores selecionados em sua pesquisa são os mais facilmente mensuráveis para a situação em que as características e a localização exata de materiais componentes são desconhecidas, especialmente nos estágios iniciais de desenvolvimento dos novos produtos.

Nota-se, ainda, de um modo geral, que os artigos usaram a literatura como fonte complementar de dados para avaliação ambiental dos processos, tanto na geração de resíduos como na produção, pela não disposição das informações nas fontes consultadas (CHEN et al., 2010; PRASARA-A; GRANT, 2011). Grande parte desses dados foi adaptada para ser usada nos países onde a pesquisa foi realizada. Alguns artigos, pela não disponibilidade de dados, citaram o problema, mas não o incluíram na avaliação ou consideraram que o item não iria interferir no resultado final, como, por exemplo: impactos ambientais gerados pelas estações de tratamento (CHEN et al., 2010) e impactos ambientais do uso de cinzas volantes e pesadas na construção de estradas (CHOWDHURY; APUL; FRY, 2010).

\section{DISCUSSÕES FINAIS}

Esta pesquisa teve o objetivo de verificar, com base nos estudos sobre a utilização de resíduos sólidos industriais provenientes de processos produtivos, como foi avaliada a variável ambiental quanto à manufatura do produto final com o uso de tais resíduos. Foram encontrados 67 artigos, dos quais a maioria (74,6\%, isto é, 50 artigos) somente mencionou de forma descritiva as possíveis vantagens que a reutilização dos resíduos poderia proporcionar, tais como, por 
exemplo, redução de impacto ambiental e de poluição, passivo ambiental, entre outros. Apesar de mencionarem as possíveis vantagens ou melhorias, não trouxeram dados concretos, como a redução de valores econômico-financeiros dos custos de produção ou a melhoria de desempenho ambiental dos produtos, que pudessem comprovar tais afirmações de forma efetiva.

Dentre os 17 artigos que avaliaram os impactos da utilização desses resíduos, oito usaram o método ACV como forma de analisar as possíveis consequências da substituição das matérias-primas originais. Desses oito artigos, somente um mencionou o tema dos custos, fator que é uma das limitações do método ACV (isto é, não considerar o fator custos na metodologia) citadas por Gäbel e Tillman (2005) e Guinée et al. (2002),

A avaliação ambiental, de um modo geral, é dependente das bases de dados existentes, do acesso a essas bases, da região geográfica onde o estudo está sendo realizado, da composição dos produtos e processos avaliados, da tecnologia, das emissões em cada fase do processo produtivo, entre outros fatores, o que provoca a grande variedade de métodos e índices avaliados. Segundo Babbit e Linder (2008b), a aplicação de diferentes métodos (Eco-indicador 99, EDIP97 e CML2001) para os mesmos dados de inventário pode originar resultados muito diferentes em cada categoria de impacto.

Verificou-se que o emprego da avaliação do ciclo de vida, por ser um método de uso mundial, é muito variável, sem ser possível definir uma estrutura uniformizada quanto à valorização de resíduos na obtenção de novos materiais. Dessa forma, muitas vezes, é difícil identificar os benefícios associados aos métodos convencionais (materiais sem o uso de resíduos) e informar o consumidor final ou usuário de um produto sobre as vantagens ambientais e as vantagens econômicas. Para as indústrias, as dificuldades persistem, pois, segundo Bovea e Gallardo (2006), um dos principais problemas do método ACV consiste em converter os resultados de uma ICV em impactos ambientais e, posteriormente, em um simples indicador ambiental que possa ser útil para os designers durante o processo de seleção dos materiais.

\section{LIMITAÇÕES}

A limitação desta pesquisa está na quantidade de artigos avaliados, motivo pelo qual os resultados não podem ser generalizados.

\section{RECOMENDAÇÕES}

A sugestão para pesquisas futuras é que, além dos períódicos, sejam consultados os artigos publicados nos congressos nacionais e internacionais sobre o tema. 


\section{REFERÊNCIAS}

ALBUQUERQUE NETO, J. R. et al. Reciclagem de pneus: uma forma de tratamento de resíduos. In: ENCONTRO NACIONAL SOBRE GESTÃO EMPRESARIAL E MEIO AMBIENTE, 8., 2005, São Paulo, Anais... São Paulo: ENGEMA, 2005. 1 CD-ROM.

ALLWOOD, J. M. et al. Material efficiency: A white paper. Resources, Conservation and Recycling, v.55, n.3, p. 362-381, 2011.

ASSOCIAÇÃO BRASILEIRA DE NORMAS TÉCNICAS (ABNT). NBR 10004: Resíduos sólidos - Classificação. Rio de Janeiro: ABNT, 2004.

ASSOCIAÇÃO BRASILEIRA DE NORMAS TÉCNICAS (ABNT). NBR ISO 14040: Gestão ambiental - avaliação do ciclo de vida princípios e estrutura. Rio de Janeiro: ABNT, 2009(a).

ASSOCIAÇÃO BRASILEIRA DE NORMAS TÉCNICAS (ABNT). NBR ISO 14044: Gestão ambiental - avaliação do ciclo de vida requisitos e orientações. Rio de Janeiro: ABNT, 2009(b).

ASSOCIAÇÃO BRASILEIRA DE NORMAS TÉCNICAS (ABNT). NBR ISO 14045: Gestão ambiental - avaliação da ecoeficiência de sistema de produto - princípios, requisitos e orientações. Rio de Janeiro: ABNT, 2014.

BABBITT, C. W.; LINDNER, A. S. A life cycle inventory of coal used for electricity production in Florida. Journal of Cleaner Production, v.13, n.9, p. 903-912, 2005.

BABBITT, C. W.; LINDNER, A. S. A life cycle comparison of disposal and beneficial use of coal combustion products in Florida. Part 1: methodology and inventory of materials, energy, and emissions. International Journal of Life Cycle Assessment, v.13, n.3, p. 202-211, 2008(a).

BABBITT, C. W.; LINDNER, A. S. A life cycle comparison of disposal and beneficial use of coal combustion products in Florida. Part 2: impact assessment of disposal and beneficial use options. International Journal of Life Cycle Assessment, v.13, n.7, p. 555-563, 2008(b).

BARBIERI, J. C. Gestão ambiental empresarial: conceitos, modelos e instrumentos. São Paulo: Saraiva, 2011.

BAUTISTA-LAZO, S.; SHORT, T. Introducing the all seeing eye of business: a model for understanding the nature, impact and potential uses of waste. Journal of Cleaner Production, v.40, p. 141-150, fev. 2013.

BERGER, M.; FINKBEINER, M. Correlation analysis of life cycle impact assessment indicators measuring resources use. International Journal of Life Cycle Assessment, v.16, n.1, p. 74-81, 2011.

BOVEA, M. D.; GALLARDO, A. The influence of impact assessment methods on materials selection for eco-design. Materials and Design, v.27, n.3, p. 209-215, 2006.

BRASIL. Decreto no 7.404, de 23 de dezembro de 2010, Regulamente a Lei no 12.305, de 2 de agosto de 2010, que institui a Política Nacional de Resíduos Sólidos - PNRS. Disponível em: $<$ http://www.trusher.com.br/_novo/areas/ documentos/lei7404.pdf >. Acesso em: 25 jul. 2013: PNRS, 2010.

BRERETON, P. et al. Lessons from applying the systematic literature review process within the software engineering domain. The Journal of Systems and Software, v.80, n.4, p. 571-583, 2007.

BRUNORI, C. et al. Reuse of a treated red mud bauxite waste: studies on environmental compatibility. Journal of Hazardous Materials, v.117, n.1, p. 55-63, 2005.

CAMPOS, L. F. A. et al. Planejamento experimental no estudo da maximização do teor de resíduos em blocos e revestimentos cerâmicos. Cerâmica, v.53, n.328, p. 373-380, 
2007.

CASTRO-GOMES, J. P. et al. Potential for reuse of tungsten mining waste-rock in technicalartistic value added products. Journal of Cleaner Production, v.25, p. 34-41, abr. 2012.

CHEN, C. et al. LCA allocation procedure used as an incitative method for waste recycling: An application to mineral additions in concrete. Resources, Conservation and Recycling, v. 54, n.12, p. 1231-1240, 2010.

CHERTOW, M. R. Industrial symbiosis: literature and taxonomy. Annual Review of Energy and Environment, v.25, p. 313-337, nov. 2000.

CHOWDHURY, R.; APUL, D.; FRY, T. A life cycle based environmental impacts assessment of construction materials used in road construction. Resources, Conservation and Recycling, v.54, n.4, p. 250-255, 2010.

CONTRERAS, A. M. et al. Comparative life cycle assessment of four alternatives for using byproducts of cane sugar production. Journal of Cleaner Production, v.17, n.8, p. 772-779, 2009.

CRAIGHILL, A. L.; POWELL, J. C. Life cycle assessment and economic evaluation of recycling: a case study. Resources, Conservation and Recycling, v.17, n.2, p. 75-96, 1996.

Di BELLA, G. et al. Modeling of perched leachate zone formation in municipal solid waste landfills. Waste Management, v.32, n.3, p. 456-462, 2012.

EKVALL, T. et al. What life-cycle assessment does and does not do in assessments of waste management. Waste Management, v.27, n.8, p. 989-996, 2007.

ESTRELA, S. P. Diagnóstico de resíduos sólidos industriais em Santa Catarina. Florianópolis, SC, 1996. 181 f. Dissertação (Mestrado em Engenharia Civil) - Universidade Federal de Santa Catarina, Florianópolis, 1996.
FIKSEL, J. et al. Comparative life cycle assessment of beneficial applications for scrap tires. Clean Technology and Environmental Policy, v.13, n.1, p. 19-35, 2011.

FLEISCHMANN, M. et al. Quantitative models for reverse logistics: a review. European Journal of Operational Research, v.103, n.1, p. 1-17, 1997.

FINNVEDEN, G. et al. Recent developments in life cycle assessment. Journal of Environmental Management, v.91, n.1, p. 1-21, 2009.

FRISCHKNECHT, R. Notions on the design and use of an ideal regional or global LCA database. International Journal of Life Cycle Assessment, v.11, Special issue n.1, p. 40-48, 2006.

FRÖHLING, M.; RETZ, O. A case study on raw material blending for the recycling of ferrous wastes in a blast furnace. Journal of Cleaner Production, v.18, n.2, p. 161-173, 2010.

GÄBEL, K.; TILLMAN, A-M. Simulating operational alternatives for future cement production. Journal of Cleaner Production, v.13, n.13/14, p. 1246-1257, 2005.

GUINÉE, J. B. et al. Handbook on Life Cycle Assessment: Operational guide to the ISO Standards. Dordrecht: Kluwer Academic Publishers, 2002.

HABERT, G.; LACAILLERIE, J. B. E.; ROUSSEL, N. An environmental evaluation of geopolymer based concrete production: reviewing current research trends. Journal of Cleaner Production, v.19, n.11, p. 1229-1238, 2011.

HODGE, M.; OCHSENDORF, J.; FERNÁNDEZ, J. Quantifying potential profit from material recycling: a case study in brick manufacturing. Journal of Cleaner Production, v.18, n.12, p. 1190-1199, 2010.

$\mathrm{HU}$, J. et al. Ecological utilization of leather tannery waste with circular economy model. Journal of Cleaner Production, v.19, n.2-3, p. 221-228, 2011. 
INTERNATIONAL ORGANIZATION FOR STANDARD - ISO 14045: 2012. Environmental management - Eco-efficiency assessment of product systems - Principles, requirements and guidelines: ISO 14045, 2012.

ITSUBO, N.; INABA, A. A new LCIA method: LIME has been completed. International Journal of Life Cycle Assessment, v.8, n.5, p. 305, 2003.

JACOBI, P. R.; BESEN, G. R. Gestão de resíduos sólidos em São Paulo: desafios da sustentabilidade. Estudos Avançados, v.25, n.71, p. 135-158, 2011.

JACOBSEN, N. B. Industrial symbiosis in Kalundborg, Denmark: a quantitative assessment of economic and environmental aspects. Journal of Industrial Ecology, v.10, n.1-2, p. 239-256, 2006.

JOLLIET, O. Emergence and future of life cycle impact assessment: good science comes from good people. International Journal of Life Cycle Assessment, v.11, Special issue n.1, p. 9-10, 2006.

JOLLIET, O. et al. IMPACT 2002+: a new life cycle impact assessment methodology. International Journal of Life Cycle Assessment, v.8, n.6, p. 324-330, 2003.

KLÖPFFER, W. The critical review of life assessment studies according to the ISO 14040 and 14044: origin, purpose and practical performance. International Journal of Life Cycle Assessment, v.17, n.9, p. 1087-1093, 2012.

LANER, D. et al. A review of approaches for the long-term management of municipal solid waste landfills. Waste Management, v.32, n.3, p. 498-512, 2012.

LEITE, P. R. Logística reversa: meio ambiente e competitividade. São Paulo: Pearson Prentice Hall, 2009.

LIAMSANGUAN, C.; GHEEWALA, S. H. LCA:
A decision support tool for environmental assessment of MSW management systems, Journal of Environmental Management, v.87, n.1, p. 132-138, 2008.

MCLELLAN, B. C. et al. Costs and carbon emissions for geopolymer pastes in comparison to ordinary portland cement. Journal of Cleaner Production, v.19, n.9/10, p. 10801090, 2011.

O'BRIEN, K. R.; MÉNACHÉ, J.; O'MOORE, L. M. Impact of fly ash content and fly ash transportation distance on embodied greenhouse gas emissions and water consumption in concrete. International Journal of Life Cycle Assessment, v.14, n.7, p. 621-629, 2009.

OGUCHI, M. et al. Fate of metals contained in waste electrical and electronic equipment in a municipal waste treatment process. Waste Management, v.32, n.1, p. 96-103, 2012.

OLIVEIRA, G. E.; HOLANDA, J. N. F. Análise do impacto ambiental causado pela utilização de resíduo sólido do setor siderúrgico em cerâmica vermelha. Cerâmica, v.50, n.315, p. 185-189, 2004.

PINSKY, V. C.; DIAS, J. L.; KRUGLIANSKAS, I. Gestão estratégica da sustentatibilidade e inovação. Revista de Administração da UFSM, v. 6, n. 3, p. 465-480, 2013.

PIZZOL, M. et al. Impacts of "metals" on human health: a comparison between nine different methodologies for life cycle impact assessment (LCIA). Journal of Cleaner Production, v.19, n.4, p. 646-656, 2011.

POTTING, J.; HAUSCHILD, M. Z. Spatial differentiation in life cycle impact assessment. A decade of method development to increase the environmental realism of LCIA. International Journal of Life Cycle Assessment, v.11, Special issue n.1, p. 11-13, 2006.

PRASARA-A, J.; GRANT, T. Comparative life cycle assessment of uses of rice husk for 
energy purposes. International Journal of Life Cycle Assessment, v.16, n.6, p. 493-502, 2011.

REDA TAHA, M. M. et al., Mechanical, fracture, and microstructural investigation of rubber concrete. Journal of Materials in Civil Engineering, v. 20, n.10, p. 640-649, 2008.

REBITZER, G. et al. Life cycle assessment Part 1: Framework, goal and scope definition, inventory analysis, and applications. Environment International, v.30, n.5, p. 701720, 2004.

STEEN, B. A. Describing values in relation to choices in LCA. International Journal of Life Cycle Assessment, v.11, n.4, p. 277-283, 2006.

TOFFOLETTO, L. et al. LUCAS - A new LCIA method used for a Canadian-specific context. International Journal of Life Cycle Assessment, v.12, n.2, p. 93-102, 2007.

VALERIO, D.; SILVA, T. C.; COHEN, C. Redução da geração de resíduos sólidos: uma abordagem econômica. In: ASSOCIAÇÃO NACIONAL DOS CENTROSDE PÓS-GRADUAÇÃO EM ECONOMIA. 36.; 2008. Salvador. Anais... ANPEC, Niterói: 2008. Disponível em: <http://www.anpec.org. br/encontro2008/artigos/200807211417570-. pdf>. Acesso em: 25 maio 2013.

WEBSTER, J.; WATSON, R. T. Analyzing the past to prepare for de future: writing a literature review. MIS Quarterly, v.26, n.2, p. xiii-xxiii, 2002.

WONG, Y. L.; LEE, K. M.; YUNG, K. C. Model scenario for integrated environmental product assessment at the use of raw materials stage of a product. Resources, Conservation and Recycling, v.54, n.11, p. 841-850, 2010.

YI, I. et al. Development of the interregional I/O based LCA method considering regionspecifics of indirect effects in regional evaluation. International Journal of Life Cycle Assessment, v.12, n.6, p. 353-364, 2007.

YANG, Q. Z. et al. Sustainable recovery of nickel from spent hydrogenation catalyst: economics, emissions and wastes assessment. Journal of Cleaner Production, v.19, n.4, p. 365-375, 2011.

YUAN, H. et al. A dynamic model for assessing the effects of management strategies on the reduction of construction and demolition waste. Waste Management, v.32, n.3, p. 521531, 2012. 
AVALIAÇÃO DE DESEMPENHO AMBIENTAL NA VALORIZAÇÃO DE RESíDUOS SÓlIDOS DE PROCESSOS INDUSTRIAIS 